\title{
Scleral buckling versus vitrectomy: can the trend be reversed suprachoroidally?
}

\author{
David Wong $^{1,2}$ - Lara Sandri ${ }^{1}$ - David H. W. Steel ${ }^{3,4}$
}

Received: 11 October 2016 / Accepted: 17 October 2016 / Published online: 16 November 2016

(C) Springer-Verlag Berlin Heidelberg 2016

Some say that younger retinal surgeons are becoming deskilled in scleral buckling (SB) surgery. Increasingly and throughout the world, the trend is to treat rhegmatogenous retinal detachments using vitrectomy $[1,2]$. Yet the consensus is that there is a continuing role for $\mathrm{SB}$ [3]; the evidence base is that some groups of patients may achieve better vision receiving primary SB rather than vitrectomy $[4,5]$. It has been suggested that the only way to reverse this trend is to make the skill set for scleral buckling more like those of vitrectomy. In this issue, El Rayes and colleagues have introduced a novel method of suprachoroidal buckling which employ techniques that might be familiar to many vitrectomy surgeons [6].

There are a host of reasons why surgeons might favour vitrectomy over SB. More and more eye surgeries are routinely carried out under local anaesthetic (LA) as day case procedures [7]. If performed under LA, scleral buckling can be more uncomfortable for the patients and thus in turn more stressful for the surgeons [8]. For routine fundal examination of patients, younger ophthalmologists are tending to use biomicroscopy instead of indirect ophthalmoscopy, reducing their skills for operating with indirect viewing. Lastly, and

David Wong

shdwong@hku.hk

1 St. Paul's Eye Unit, Royal Liverpool University Hospital Trust, Liverpool, UK

2 Graefe's Archives of Clinical and Experimental Ophthalmology, St. Paul's Eye Unit, Royal Liverpool University Hospital Trust, Liverpool, UK

3 Sunderland Eye Infirmary, Queen Alexandra Road, Sunderland, UK

4 Institute of Genetic Medicine, Newcastle University, Newcastle Upon Tyne, UK perhaps most importantly, detailed preoperative examination is time-consuming, and the temptation to rely on vitrectomy with "internal search" to identify retinal breaks and treat as seen using the internal approach is strong [9].

Mikhail et al. (6) described the use of endo-illumination with a chandelier light source during suprachoroidal buckling to allow internal searching to identify and localise retinal breaks, and used the wide-angle optics of the operating microscope instead of indirect ophthalmoscopy. The French pioneering retinal surgeon Madam Bonnet wrote extensively about this technique back in the 1970s, and she even achieved laser retinopexy via the operating microscope without entering the eye [9-12]. In 2016, Narayanan et al. pointed out that SB using endoillumination enabled the trainer to see exactly what the trainee sees, and as such the technique could have additional educational and supervisional merit [13]. Suprachoroidal buckling crucially takes one further step forward. No rectus muscles traction sutures seemed to be necessary. This could avoid much of the discomfort associated with scleral buckling. Finally, refractive changes associated with scleral buckling may be avoided, and there seems to be no reason why external drainage combined with an infusion could not be used if needed to reduce the risk of drain haemorrhage. Indeed, the technique of suprachoroidal buckling could, however, be said to be more akin to vitrectomy (using an internal approach) than to SB (using an external approach).

The results of Mikhail et al. (6) are impressive; however, the safety and efficacy of suprachoroidal buckling requires corroboration from other centres. Indeed, there are still many questions. The authors mention a steep learning curve but don't mention the obstacles met on this. Large breaks can be treated but encirclement is not possible. Healon 5 (Abbott Medical Optics, Santa Ana, CA, USA) as used, gives a buckling effect for $\sim 2-3$ weeks: but is this long enough for cases with very chronic subretinal fluid, a common feature of many 
cases considered for buckling? There were no cases of significant choroidal haemorrhage, which is perhaps surprisingespecially as cryotherapy was applied in $61 \%$ of the cases before buckling. The authors used a fine flexible illuminated cannula, and attribute their low haemorrhage rate partly to this. A new olive-tipped rigid cannula specifically made for the procedure is now available however (Olive Tip SC Cannula; Medone, Sarasota, FL, USA) and the side-effect profile of this is unknown. What happens when the break overlies or lies immediately behind a vortex vein? The authors recommend avoiding contact with these, but their position can be difficult to ascertain with bullous detachment. The procedure is quick, but then again so is a single radial scleral buckle in the right case.

The evidence base already suggests to us that we should be performing more $\mathrm{SB}$, and if we did we might achieve better visual outcomes for our patients [4, 5]. Myopic retinal detachment secondary to atrophic round holes and without posterior vitreous detachment can be treated primarily with SB with excellent anatomical and visual results [14]. Certainly, few would argue that most retinal detachment arising from retinal dialysis should be buckled, especially in children and young adults [15]. Knowing and doing, however, are two separate things. It is unrealistic to think that a new generation of retinal specialists will suddenly revert to using indirect ophthalmoscopy and applying SB in the conventional way. Potentially, if the results of Mikhail et al. are corroborated, this new innovative approach could bridge the gap between these two disparate approaches. Furthermore, SB and vitrectomy need not be mutually exclusive with this new technique as in conventional SB. There is evidence to suggest that in cases of retinal detachment associated with a high risk of proliferative vitreoretinopathy, a combination of vitrectomy and scleral buckling may be more effective than vitrectomy alone [16].

In the current era of vitrectomy for most, if not all, retinal detachments, there should still be a place for buckling surgery - reinventing it may be the way to do this. If nothing else, revival in the interest of a buckling approach may make surgeons spend a little bit more time examining the retina preoperatively, and this alone might have a positive effect on surgical outcomes.

\section{References}

1. Ramulu PY, Do DV, Corcoran KJ et al (2010) Use of retinal procedures in medicare beneficiaries from 1997 to 2007. Arch Ophthalmol 128:1335-1340

2. Gupta B, Neffendorf JE, Williamson TH (2016) Trends and emerging patterns of practice in vitreoretinal surgery. Acta Ophthalmol [Epub ahead of print]. doi:10.1111/aos.13102

3. Ryan EHJ, Mittra RA (2010) Scleral buckling vs vitrectomy: the continued role for scleral buckling in the vitrectomy era. Arch Ophthalmol 128:1202-1205

4. Heimann H, Bartz-Schmidt KU, Bornfeld N et al (2007) Scleral buckling versus primary vitrectomy in rhegmatogenous retinal detachment. a prospective randomized multicenter clinical study. Ophthalmology 114:2142-2154

5. Wong CW, Yeo IYS, Loh BK et al (2015) Scleral buckling versus vitrectomy in the management of macula-off primary rhegmatogenous retinal detachment: a comparison of visual outcomes. Retina 35:2552-2557

6. Mikhail M, El Rayes EN, Kojima K et al. (2016) Catheter-guided suprachoroidal buckling of rhegmatogenous retinal detachments secondary to peripheral retinal breaks. Graefes Arch Clin Exp Ophthalmol [Epub ahead of print]

7. Sallam AA, Donachie PH, Williamson TH et al (2016) The Royal College of Ophthalmologists' National Ophthalmology Database Study of vitreoretinal surgery: report 5 , anaesthetic techniques. $\mathrm{Br}$ J Ophthalmol 100:246-252

8. Rao GP, Wong D, Groenewald C et al (1998) Local anaesthesia for vitreoretinal surgery: A case-control study of 200 cases. Eye 12: 407-411

9. Bonnet M (1975) Advantages of surgery under the microscope in treatment of detachment of the retina. Bull Soc Ophtalmol Fr 75: $1111-1112$

10. Bonnet M (1979) Biomicroscopy of the fundus in retinal detachment surgery (author's transl)]. J Fr Ophtalmol 2:209-216

11. Bonnet M, Grange JD (1979) Functional results of 100 successive cases of retinal detachment operated on under microscope. Bull Soc Ophtalmol Fr 79:21-24

12. Bonnet M (1980) Argon laser adapted on the surgical microscope (author's transl). J Fr Ophtalmol 3:127-128

13. Narayanan R, Tyagi M, Hussein A et al (2016) Scleral buckling with wide-angled endoillumination as a surgical educational tool. Retina 36:830-833

14. Ung T, Comer MB, Ang AJS et al (2005) Clinical features and surgical management of retinal detachment secondary to round retinal holes. Eye (Lond) 19:665-669

15. Errera M-H, Liyanage SE, Moya R et al (2015) (2015) Primary scleral buckling for pediatric rhegmatogenous retinal detachment. Retina 35:1441-1449

16. Storey P, Md MPH, Alshareef R et al (2014) Pars plana vitrectomy and scleral buckle versus pars plana vitrectomy alone for patients with rhegmatogenous retinal detachment at high risk for proliferative vitreoretinopathy. Retina 34:1945-1951 